\title{
$-\quad 167-$
}

ftem bes Rủénmarls angreift, convulfipifde Berwegun, gell, grofe Unregelmípigeteten in ben Funtionen ber Res fpiration unb Eirculation erfolgen. 2lüerbem zeigt fid, Daß es birett bie Euntractibilitat uno Jrritabilitát bee

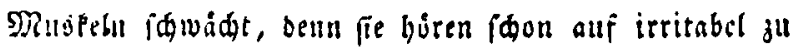

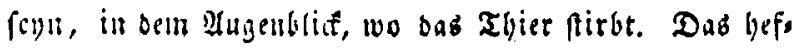
tige Erbeeden, weldpes fiets fratt finbet, felbft wenn eine blope Injection in bas Zellgenebe gemawt worben iff, jeigt, Duf bet Nagen entweder direlt oder ourd Mitleis Demidaft you bem Giffe afficitt wirb.

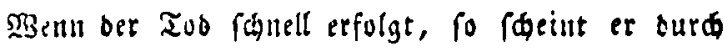

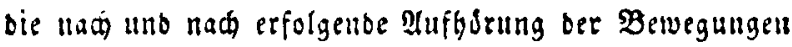
bes Sherjens und ber Refpiration zul erfolgen, wenn aber bas Eeben no einige Beit nad ber 2 upnabme bed Gifted im Plagen fortbauert, fo fdeitut ber Tob in Folge ciner intenfiven Entzůnbung ber Braftros Snteftinal= Eøleims bállte ju erfolgen.

Bei der Behandlung einer Bergiftung biefer IItt mus man juerft bie finnelle Entleerung bes Biftes ju bes

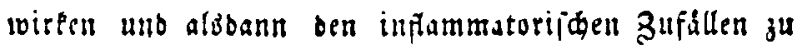
begegnen fuden. Zu bemerten ift nod, bas bab Enveis bier Peine Dienffe leiffet, wie beim Sublimnt, woib es bas Eyanio nidat jeriegrt, fo saß man es bier nur als cin linsernbes Mittel betraden Pann (Journal de Chim. med. I, 222).

\section{Sdinefat unb Sutedifther.}

Bir baben bereitb friber ber Bercirung bes Aethiops Swwefets mineralis gedadt unb ber Ifngaben yon $\mathfrak{Z} a$ bocy, Auethilibser, 


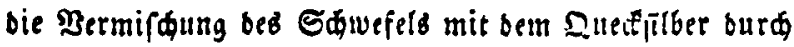

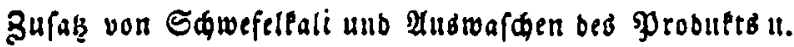
f. w. ju befsebern, fo wie ber son (B)eiger, hybrothions faures Ammouial ftatt bes હdivefelfalis zu nchmen, wos burd bas 2fustwafdell vermieben werbe. Szert $\mathfrak{T} \mathfrak{q}$. $\mathfrak{R}$ a $\boldsymbol{r}_{\mathrm{s}}$ ti us madte biegegen bie Bemerlung, baß ber fo barges fellte Aethiops Almmonial enthalten msdate. Sgetr of eis

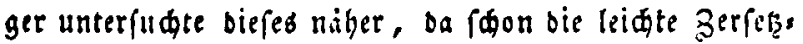
barteit bes gndrothionfauren 2 fmmoniafs es ihm nidt

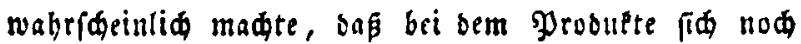
Ammonial befinden wiloc. Diefes ergab aud bie unter: fudung. Nur wenn die Jugrebienjien nidt rein fint, ber Sdinefel ober bab hobrotbionfaure Mmmonial Sdwes felfätre enthalten, wiro ber Aethiops ettwas IImmoniaf, burd bie Berbintoung nit biejer Salure, beigemi/dt

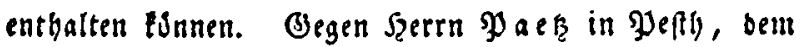
(3) eiger's Berfabren nide gefingen wollte, fand F̧err (3., baß biefer nidt gebdrig mujfe gearbeitet baben, weil

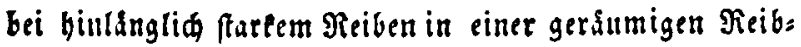

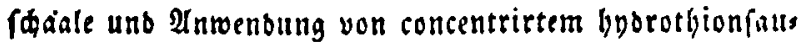
ren Anmonial ihm fetb biefé Berfabren ein befriedigens bes Refultat gab. Der burd Reiben bereitete Aethiops,

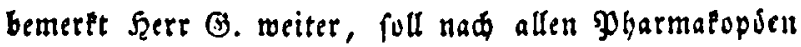

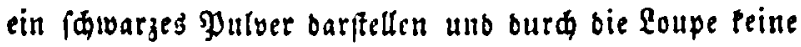
Quecefilbertbeilden mebe ertennen laffen. Birb er aber mit $\mathbb{B a f f e r}$ angerieben, fo werben fid visle Duedfilbers Pligeldben albjondern.

Reine Yुrufung fdreibt yor, ben Aethiops auf fol: we Beife ju unterfuden, unb ed milfe baber berfelbe als gut alngefelyen werben, wenn er trode nteine Quedfil: 
berlígelden mebr zeige, fo dap er ein Bemenge von Sdivefelquedfilber coser hyorothionfaurm Quecifibers

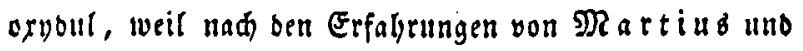

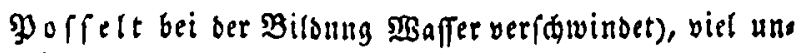
veriunbenen Shwefel uno getsotetem Quefillber i if.

Der nad Taboeys Derfabren burd Sdrefeleali und 2Auswarden mit Saffer bereitete Aethiops Paun teili frimjertheiltes metallifaes Dueafifiber mebr entbals

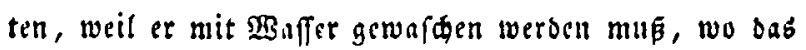
Metall feine laufende (3efralt wicber anneǵmen wirbe.

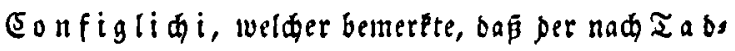
bev) bargeftellte Aethiops, wahrfheintid wegen einen (jes

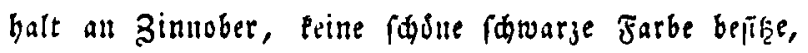
fdlug vor, fdrwefelfaures Dueffiliber mit Baffer zu was fhen, ben erbaltenen Turpith mit gleidem Gewidt Sdires felblumen unter 3ufas yon etwas હdwefelfalilsfung unb Erivämen zu reiben, anb̧umafden unb zu trof́nen. Diefer Aethiops wuirbe aber nidt nur meḩr Sdwefel entlyalten als ber geroshnticte, fonbern (3) i ger n gelaltg es alld nidt, allf biefem $\mathfrak{B}$ ege einen fabinen fduarzen Aethiops onrjuffellen; er befam Gemenge, bie eine roths geibe Farbe befapen unb aus zinnober unb Edivefel bes fanten, unb uad 2luswnfdjen und Troffnen ein afdgraues

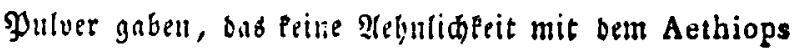
batte (3) eiger's Magajia IX. 5n).

\section{$\mathfrak{B} \mathfrak{l} \mathfrak{e} \mathfrak{l}$.}

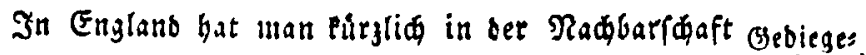

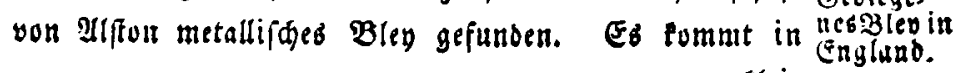




\section{$-170-$}

fleiten runbliden פRaffen, von Bleyglanz, Bleyglátte, Blinde und Quard begleitet, in Moern in Snltftein vor. Die gauje Maffe biefer abeen foeint febr jerferzt zu feyn, uub dus פRetall ift mit eiluem weißen mehligen Befdlag ron fálecfelfaurem Bglen bebeft (Edinb. Journ. of Science, Annals of Philos. N. S. 1X. 154).

Mlenges balt in Epeiien.

Heber den Bleggebalt ber Epeifen, weun folde in jimnernen bleybaltigen Oefispen bercitet merben, bat frers Dr. (J) i ger (3) Magaj. X.116) lehe belebre:be Unterfu, dungen mitgethrilt, welaje in geeigneten zallen $\mathfrak{R}$ ufmerts

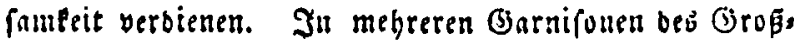
letjogthumb Baben beftunden Dampflowapparate jut 3. bereitung ber Speifen fir die Eoldaten. Die Sudjacs

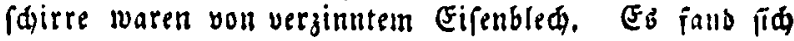
nber, bas bie meifen Soloaten bie allf bicfe צ̧acife jus bereiteten Spcifan nide vertragen Ponnten, fonbern uad einiger ふacit Manget an slppetit, Epet, Leib/dmerjen u. f. w. betamen. Diefe anfalten gingen desbalb fpiter sin. Serr Dr. Bjeiger unterfudte sie in siefen Slppas raten bercitete Fleijabriffe. Sie entblelt felbe garinge Epuren von 3iun, unb vielleidt all MRinima von $\mathfrak{B l e y , ~}$ die Sartoffelfuppe sebglciden. Dnd Fett bes Dampfers:

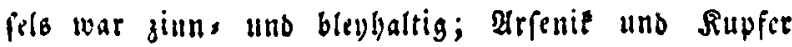
puntte man aber nidt barin entbeden. Es gejt bieralts beutlid herwor, bas bie in verjinnten Eifenbled bereites ten Epcifon Epuren von Zinn, vielleidt aud von Bley ents balten einnen. Db bie geriatge Menge beffelben nudtbeis

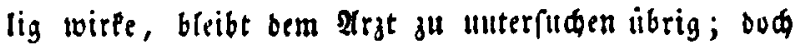
modere tallm in atbrebe geffellt werben esnnen, baß ber

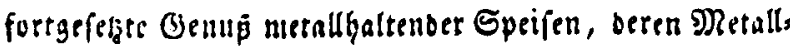


gefalt nad Maafgabe ber Umftánbe balb geringer, balb grop̧er fegn fann, allf die Dauer naátheilig mirfe. Das Fett in ben Dampfeffiel und ben ઉefásen funnte beshalb reider an Sinn: uno Blengehalt fenn, weil burd die

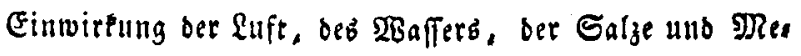
talloxyce, mie burd Mlfalien bei ber Eeifenbiloung, bafa felbe ju Del s unb Falgfäure verdubert toiro, zumal weun niđt getsjrige Reinigung uno Aht famfrit auf alle bie Umftäne gegeben wirb, sie folde Einwirtung begúufti. gen Fisnen.

\section{Siley und Sauerftor.}

Die bisherigen פRetluben, bie Bleyalde ju rebucis giebuttion

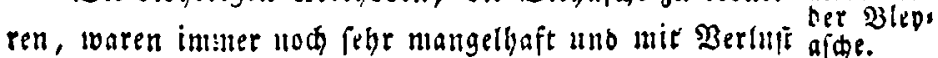
verbunben, nud wurbe bei mebreren berfetten bab $\mathfrak{B l e y}$ fo fefi, on zur Bleylveiffabrifation, nidt melgr eignete, mešbalb

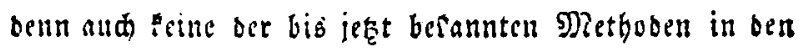
Bleyweiffabritu 2tnecroung faub, unb cine grope Mens

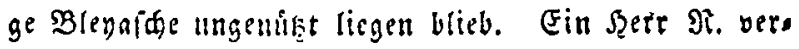

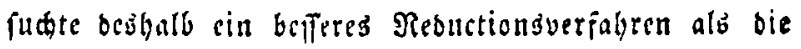
bisherigen, bus Rebliciren in Dpen mit Flammenfeuer, theils in Eupulo: Deen mit Dlababigen unb Rohlen, theils

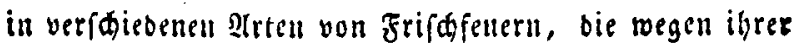
gropen Shiz̧e, die fie erforbern, felfe viele Radtbeile bas ben, uno erreidte feinen 3̧wet baburd, baß er in einem befuntern cifernen $\mathfrak{D}$ fen die $\mathfrak{B l e y a f d e ~ m i t ~ b e n ~ l o g e n a n n s ~}$

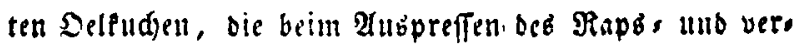
wanter Saamen als Ritafifnde binterbleiben, glibete.

Die 